\title{
A new two-pole accretion polar: RX J1846.9+5538`
}

\author{
R. Schwarz ${ }^{1}$, J. Greiner ${ }^{1,2}$, G. H. Tovmassian ${ }^{3}$, S. V. Zharikov ${ }^{3}$, and W. Wenzel ${ }^{4}$ \\ 1 Astrophysical Institute Potsdam, An der Sternwarte 16, 14482 Potsdam, Germany \\ 2 Max-Planck-Institut für Extraterrestrische Physik, Giessenbachstraße, 85740 Garching, Germany \\ 3 Instituto de Astronomía, UNAM, Apdo.Post. 877, 22860 Ensenada, B.C., México \\ 4 Sonneberg Observatory, 96515 Sonneberg, Germany \\ Received 9 April 2002 / Accepted 29 May 2002
}

\begin{abstract}
We report the discovery of a new, bright $\left(V \sim 17^{\mathrm{m}}\right)$ AM Her system as the optical counterpart of the soft ROSAT All-Sky-Survey source RX J1846.9+5538 (=1RXS J184659.4+553834). Optical photometric and spectroscopic follow-up observations reveal a single period of $128.7 \mathrm{~min}$, consistent with a high degree of spin-orbit synchronization, and a low polar field strength $(B<20 \mathrm{MG})$ of the primary accretion region. The system was observed in optical intermediate and high states that differ by about $1 \mathrm{mag}$. These brightness variations were accompanied by a correlated change of the optical light curve, which we interpret as a switch between one- and two-pole accretion. This explanation is also supported by the X-ray light curves, which at two different epochs display emission from two equally bright accretion regions separated by $\sim 160^{\circ}$. Both spots possess distinct spectral X-ray properties as seen from the X-ray hardness ratio, where the secondary accretion region appears significantly softer, thus probably indicating a higher field strength compared to the primary region. In all ROSAT pointings a deep dip is present during the primary flux maxima, very likely caused by absorption in one of the accretion streams.
\end{abstract}

Key words. X-rays: stars - cataclysmic variables - accretion - stars: magnetic fields - stars: individual: RX J1846.9+5538

\section{Introduction}

AM Her type variables are a subgroup of cataclysmic variables in which the magnetic field of the white dwarf controls the geometry of the material flow between the main-sequence donor and the white dwarf primary (see e.g. Warner 1995 for a detailed review). The inflow of matter along the magnetic field lines (of one or occasionally also two magnetic poles) is decelerated above the white dwarf surface producing a shock front. This region is thought to emit hard X-rays (usually modelled in terms of thermal bremsstrahlung of 10-20 keV) and polarized cyclotron radiation (hence these systems are also named polars) in the IR to UV range. In addition, a strong soft component has been frequently observed from polars that is thought to arise from the heated accretion pole (usually modelled in terms of a blackbody of 20-50 eV).

It is this soft X-ray component which has led to the discovery of a few dozen new polars by ROSAT observations over the last decade, most notably the ROSAT all-sky survey (Beuermann \& Burwitz 1995). The source described here has been discovered as a result of a systematic survey for supersoft X-ray sources from the all-sky survey data (Greiner 1996 for

Send offprint requests to: R. Schwarz, e-mail: rschwarz@aip.de

* Partly based on observations collected at the GermanSpanish Astronomical Centre, Calar Alto, operated by the MPI für Astronomie, Heidelberg, jointly with the Spanish National Commission for Astronomy. details of this survey) which revealed a large number of CVs and single white dwarfs. Other confirmed polars identified from this sample include V844 Her = RX J1802.1+1804 (Greiner et al. 1995, 1998), RS Cae = RX J0453.4-4213 (Burwitz et al. 1996) and V1007 Her $=$ RXJ1724.0+4114 (Greiner et al. 1998).

In this paper we present photometric, spectroscopic and X-ray observations (summarized in Tables 1 and 2) that led to the discovery of a new polar, RX J1846.9+5538 (henceforth referred to as RX J1846).

\section{ROSAT X-ray observations}

\subsection{All-Sky Survey and optical identification}

RX J1846 was scanned during the ROSAT All-Sky-Survey (RASS) over a period of 8 days in October 1990 for a rather long (due to its high ecliptic latitude) total observing time of 1610 s. Its mean count rate in the ROSAT position-sensitive proportional counter (PSPC) was $0.2 \mathrm{cts} / \mathrm{s}$, and the hardness ratio $H R 1=-0.85 \pm 0.06$, where $H R 1$ is defined as $(\mathrm{H}-\mathrm{S}) /(\mathrm{H}+\mathrm{S})$, with $\mathrm{H}(\mathrm{S})$ being the counts above (below) $0.4 \mathrm{keV}$ over the full PSPC range of $0.1-2.4 \mathrm{keV}$.

Within the X-ray positional error circle (see Fig. 1) there are two bright optical sources, USNO A2.0 1425.09302300 (named Star A in the finding chart) and $12^{\prime \prime} \mathrm{SW}$ of that, the cataclysmic binary identified spectroscopically (see Sect. 4). 


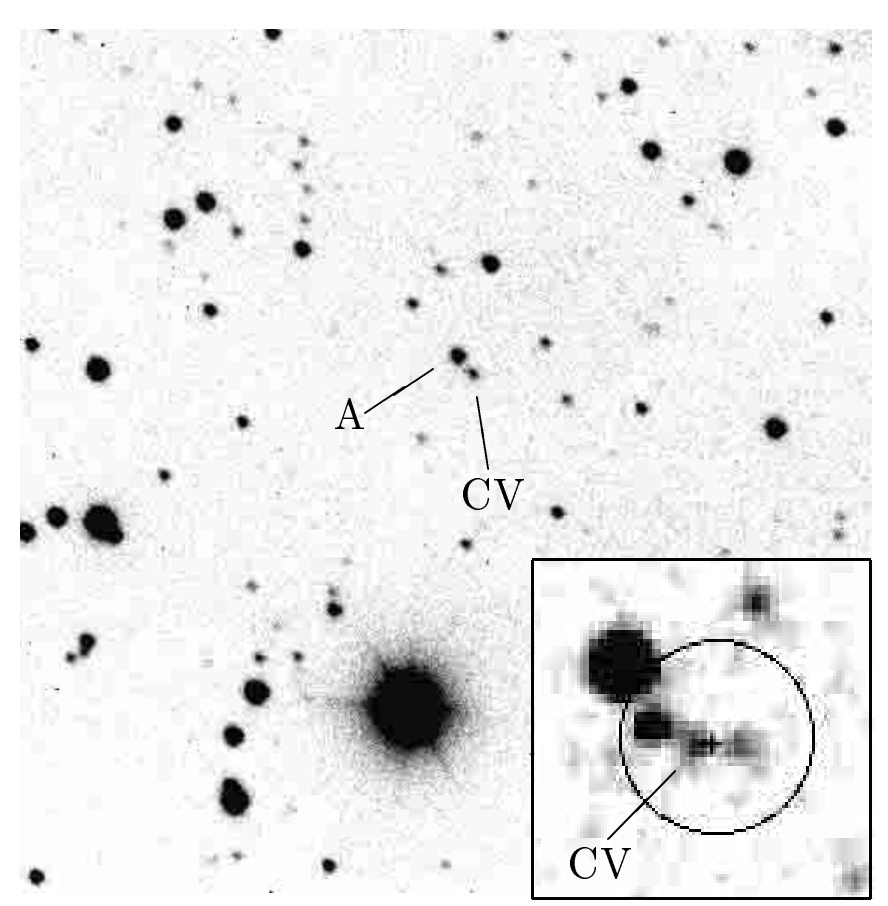

Fig. 1. White-light CCD image of RX J1846.9+5538 obtained with the AIP $70 \mathrm{~cm}$ telescope. North is top and East to the left. The size of the field is approximately $8^{\prime} \times 8^{\prime}$. The inset at the lower right corner shows a 30" $\times 30^{\prime \prime}$ blow up taken from the digitized Palomar Observatory Sky Survey (POSS) 2 together with the $2 \sigma$ X-ray error circle derived from the HRI pointing. The bright star in the upper left of the blow up is star A. The cataclysmic variable RX J1846.9+5538 was certainly in an optically low state during the POSS exposure. Its optical position was determined to $\alpha_{2000}=18^{\mathrm{h}} 46^{\mathrm{m}} 58^{\mathrm{s}} .9$ and $\delta_{2000}=55^{\circ} 38^{\prime} 29^{\prime \prime}\left( \pm 1^{\prime \prime}\right)$.

Its optical position is measured as: RA $(2000.0)=18^{\mathrm{h}} 46^{\mathrm{m}} 58^{\mathrm{s}} .9$, $\operatorname{Dec}(2000.0)=55^{\circ} 38^{\prime} 29^{\prime \prime}\left( \pm 1^{\prime \prime}\right)$. Inspection of the digitized Palomar Observatory Sky Survey 2 plates taken in October 1991 (see blow-up in the lower right corner of Fig. 1) revealed two additional fainter sources 5" east and west of $\mathrm{RX} \mathrm{J} 1846$. At that time the $\mathrm{CV}$ had dropped into a deep low state of $\sim 20^{\mathrm{m}}$.

\subsection{X-ray light curves}

For the timing analysis the RASS photons were extracted within a radius of $4^{\prime}$ around the X-ray position of RX J1846. The background was chosen at the same ecliptic longitude at $\sim 1^{\circ}$ distance, corresponding to the photons detected typically $15 \mathrm{~s}$ before or after the arrival of the source photons. Standard corrections were applied using the dedicated EXSAS software package (Zimmermann et al. 1994) including barycenter correction.

Further dedicated follow-up pointed ROSAT observations were performed on June 15-18, 1992 with the PSPC and on April 8-13, 1995 with the high-resolution imager (HRI). In addition, RX J1846 was also covered in a serendipitous pointing on September 25/26, 1993 at about $37^{\prime}$ off-axis angle. Using the EXSAS package, source photons were extracted with a radius of $1.5,7.5$ and 0.8 for the on-axis PSPC pointing, the off-axis PSPC pointing and the HRI pointing, respectively.
Table 1. Log of optical observations.

\begin{tabular}{lllrr}
\hline \hline Telescope & Date & $\begin{array}{l}\text { Range } \\
(\AA / F i l t e r)\end{array}$ & $\begin{array}{r}T_{\text {Dur }} \\
(\mathrm{hrs})\end{array}$ & $\begin{array}{r}T_{\text {Int }} \\
(\mathrm{s})\end{array}$ \\
\hline \multicolumn{5}{c}{ spectroscopy } \\
CA $3.5 \mathrm{~m}$ & 1992 Oct. 1 & $3900-7200$ & 1.0 & 3600 \\
SAO $6.0 \mathrm{~m}$ & 1999 Jun. 19 & $3400-7900$ & 3.6 & 900 \\
\multicolumn{5}{c}{ photometry } \\
SO 0.6 m & 1994 Sep. 21 & $R$ & 4.1 & 600 \\
SO 0.6 m & 1994 Sep. 22 & $R$ & 3.7 & 600 \\
SO 0.6 m & 1994 Sep. 23 & $R$ & 2.2 & 600 \\
SO 0.6 m & 1994 Sep. 25 & $R$ & 0.5 & 600 \\
SO 0.6 m & 1994 Sep. 27 & $W L$ & 0.9 & 300 \\
SO 0.6 m & 1994 Sep. 29 & $W L$ & 4.8 & 180 \\
SO 0.6 m & 1994 Oct. 12 & $R$ & 1.3 & 600 \\
SO 0.6 m & 1994 Oct. 13 & $W L$ & 0.4 & 600 \\
SO 0.6 m & 1994 Oct. 14 & $W L$ & 0.3 & 600 \\
OAN 1.5 m & 1995 May 2 & $W L$ & 3.2 & 120 \\
OAN 1.5 m & 1995 May 4 & $W L$ & 2.6 & 90 \\
AIP 0.7 m & 1995 Oct. 24 & $W L$ & 2.3 & 120 \\
AIP 0.7 m & 1997 Oct. 28 & $W L$ & 1.3 & 60 \\
AIP 0.7 m & 1998 Jan. 25 & $W L$ & 4.7 & 30 \\
AIP 0.7 m & 1998 Mar. 24 & $W L$ & 3.7 & 60 \\
AIP 0.7 m & 1998 Aug. 5 & $W L$ & 3.3 & 60 \\
AIP 0.7 m & 1998 Sep. 24 & $W L$ & 2.3 & 60 \\
\hline
\end{tabular}

The abbreviations have the following meaning: $\mathrm{CA}=$ Calar Alto, $\mathrm{SAO}=$ Special Astrophysical Observatory, Zelenchukskaja, $\mathrm{SO}=$ Sonneberg Observatory, OAN = Observatorio Astronómico Nacional de San Pedro Mártir, AIP = Astrophysical Institute Potsdam, $W L=$ white light

Table 2. Log of the X-ray observations of RX J1846.9+5538.

\begin{tabular}{crrcc}
\hline \hline Date $^{1}$ & $T_{\text {Exp }}$ & $N_{\text {cts }}$ & $\begin{array}{c}\text { Mean rate } \\
\text { (cts/s) }\end{array}$ & $H R 1$ \\
\hline 1990 Oct. 12-19 P & 1610 & 136 & 0.12 & -0.85 \\
1992 Jun. 15-18 P & 10540 & 2478 & 0.28 & -0.74 \\
1993 Sep. 25/26 P & 27510 & 1895 & 0.10 & -0.60 \\
1995 Apr. 08-13 H & 17950 & 280 & $0.02^{2}$ & - \\
\hline
\end{tabular}

${ }^{1}$ The letters after the date denote the ROSAT detector: $\mathrm{H}=\mathrm{HRI}, \mathrm{P}=$ PSPC.

${ }^{2}$ Note the lower sensitivity of the HRI at soft energies by a factor of 7.8 .

The background was chosen from concentric circles around the source region with radii of $3^{\prime}, 12.5$ and $2^{\prime}$, respectively. Other nearby sources were cut out, and the background area normalized to the source extraction area before the background subtraction. Table 2 summarises these measurements.

The RASS light curve folded over the photometric ephemeris as derived in Sect. 3.1 is shown in Fig. 2 (upper panel). It displays a $100 \%$ modulation with a peak count rate of nearly $1 \mathrm{cts} / \mathrm{s}$ and a pronounced faint-phase where the X-ray flux is practically zero (formal count rate of 


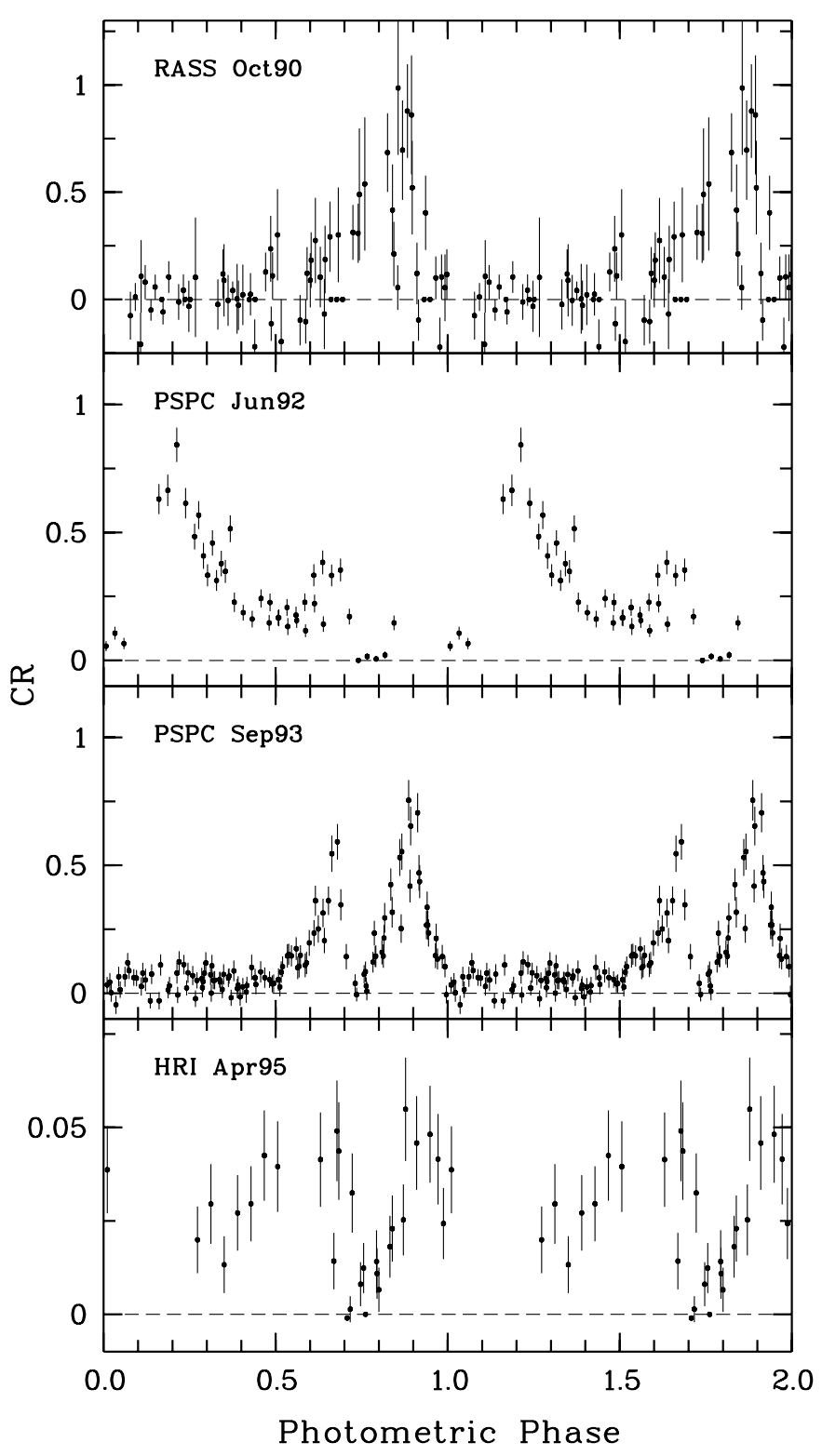

Fig. 2. ROSAT X-ray light curves of RX J1846.9+5538.

$-0.006 \pm 0.10 \mathrm{cts} / \mathrm{s})$. This "on-off" profile is typical for polars where the emission region disappears behind the limb of the white dwarf for part of the orbit. The short duration of the bright phase, which lasts only 0.3 of the orbit, requires the main accretion region to be in the lower hemisphere of the white dwarf. A similar morphology was also seen during the longest pointed observation in September 1993, but now with a bright phase extended to $\Delta \phi \sim 0.5$. The phasing of that X-ray bright phase strongly suggests that it is due to the same primary accretion region seen also in the optical light curves during the intermediate state in 1997/98. The PSPC observation in September 1993 reveals a non-zero count rate $(0.048 \pm 0.002)$ also during the faint phase.

This simple "bright-faint" behaviour was fundamentally altered in the June 1992 PSPC pointing. Most strikingly, we observe the X-ray flux peaking at $\phi=0.23$, the phase of the former faint interval. Although the rise to maximum is not covered and the decline probably overlaps with emission from the still active primary region, the shape of this hump is suggestive of emission from a second self-eclipsing accretion region. This is even more convincingly confirmed by the spectral softening during the flux peak (see Sect. 2.3). This secondary accretion spot is equally bright compared to the primary one, reaching a count rate of $0.8 \mathrm{cts} / \mathrm{s}$.

Further HRI observations were carried out in April 1995, which together with the photometry obtained three weeks later provide the closest X-ray/optical observations in time. The lack of complete orbital coverage and spectral resolution render these observations not completely conclusive. At that time the system was also X-ray bright at phase 0.3 , which might be interpreted as two-pole accretion, probably seen also later in the optical. On the other hand, the flux peak at phase 0.25 as seen in June 1992 is missing, and the light curve can also be explained as a result of a prolonged primary bright phase.

All pointed observations show, contrary to the RASS observation, a marked decrease of the X-ray flux in the bright phase at around $\phi \sim 0.7$. During the 1993 PSPC and the 1995 HRI observation the dip had a short ingress, and was followed, after a short interval $(\Delta \phi<0.01)$ of totality, by a rather protracted egress. The duration of the dip at these occasions, from start to complete recovery of the pre-dip level, lasted 0.19 phase units. The shape of the dip was considerably altered in 1992, when it was more flat-bottomed and the X-ray flux was zero for about $\Delta \phi \simeq 0.07$. Dip egress was insufficiently covered in this pointing. The occurrence of such dips in polars is in general understood as a consequence of photoelectric absorption by matter of the accretion stream. The X-ray spectral hardening during dip phase and the subsequent softening during egress (see Sect. 2.3) are in line with the expected energy dependence of cold absorption. Possible alternative explanations, like an eclipse by the secondary or a highly structured accretion region, are not consistent with the phase variability and the sharp ingress/egress of the dip observed in RX J1846. In context with the photoelectric absorption, the modification of the dip profile in the 1992 PSPC observation would imply a denser and more collimated stream, supposedly connected with the increased activity of the secondary pole at that time.

\subsection{X-ray spectroscopy}

The two pointed PSPC observations have resulted in the detection of more than 4400 photons, thus enabling spectral investigations along the orbital period. As a first step we used the hardness ratio $H R 1$ (as defined in Sect. 2.1) which is shown as a function of the orbital period in Fig. 3. It is highly variable, changing from 0.5 to -1 depending on which accretion region was visible. For the primary accretion region that was covered during both pointings the $H R 1$ was consistently at an intermediate value of -0.57 . Contrary to that, the secondary pole active only in June $1992(\phi=0.1-0.35)$ is supersoft with $H R 1=-0.92$. For the faint phase observed in the 1993 pointing the $H R 1$ changes between -0.5 to 0.5 with an average value of -0.11 , notably harder than the two main emission regions.

The average X-ray spectrum of RX J1846 is well represented by a two component model consisting of a blackbody 

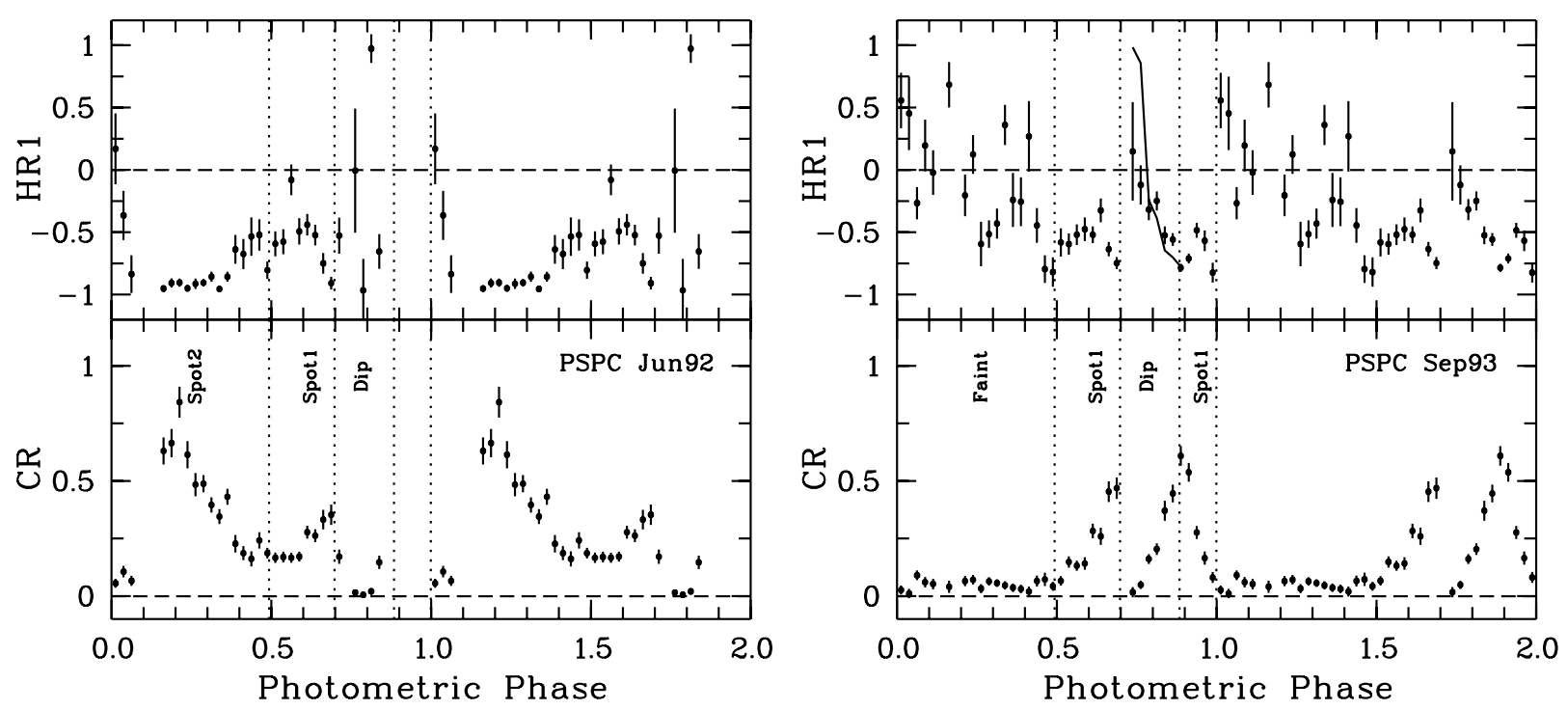

Fig. 3. Hardness ratio variations (upper panel) of RX J1846 during the pointed ROSAT observation in June 1992 (left) and September 1993 (right). In the lower panel the corresponding X-ray light curves are shown. Dotted vertical lines indicate the beginning and the end of the primary bright phase and the absorption dip in the 1993 data. The solid line in the upper right panel shows the predicted variation of $H R 1$ in the absorption dip.

Table 3. Fit results for phase-resolved X-ray spectra.

\begin{tabular}{lclcc}
\hline \hline Data & $\begin{array}{c}N_{\mathrm{H}} \\
\left(10^{20} \mathrm{~cm}^{-2}\right)\end{array}$ & $\begin{array}{l}F_{\mathrm{bb}} \\
\left(10^{-12} \mathrm{erg} / \mathrm{cm}^{2} / \mathrm{s}\right)\end{array}$ & $\chi_{\text {red }}^{2}$ \\
\hline 92 spot1 & $1.3 \pm 0.7$ & 6.5 & 5.0 & 0.27 \\
93 spot1 & $1.2 \pm 0.5$ & 6.8 & 4.2 & 0.51 \\
92 spot2 & $0.8 \pm 0.3$ & 9.9 & 2.0 & 2.13 \\
93 faint & $0.7 \pm 1.6$ & 0.5 & 1.7 & 1.13 \\
\hline
\end{tabular}

The blackbody and bremsstrahlung temperatures have been fixed at $k T_{\mathrm{bb}}=25 \mathrm{eV}$ and $k T_{\mathrm{br}}=20 \mathrm{keV}$ during the fit. The total galactic absorbing column is $4.9 \times 10^{20} \mathrm{~cm}^{-2}$ (Dickey \& Lockman 1990).

plus a thermal bremsstrahlung model having a low absorption $\left(N_{\mathrm{H}}<10^{21} \mathrm{~cm}^{-2}\right)$ and wide range of possible blackbody temperatures $15-40 \mathrm{eV}$ (Fig. 6). Since the temperatures of both components are not well constrained by the ROSAT data and the photon statistics of the phase-resolved spectra are low, we concentrate in the following on the determination of the $\mathrm{X}$-ray fluxes assuming fixed values for the temperatures, set to $k T_{\mathrm{bb}}=25 \mathrm{eV}$ and $k T_{\mathrm{br}}=20 \mathrm{keV}$. Spectra from the different emission regions were extracted separately using the phase intervals 0-0.4 (spot2 1992), 0-0.492 (faint phase 1993) and $0.492-0.697,0.492-0.697$ for the primary accretion region. The resulting best-fit values of the absorption and the unabsorbed, bolometric blackbody and bremsstrahlungs fluxes are summarized in Table 3.

As already indicated by the count rates and hardness ratio, both accretion regions emit the same total X-ray flux of $F_{\mathrm{X}} \sim 1 \times 10^{-11} \mathrm{erg} / \mathrm{cm}^{2} / \mathrm{s}$, but have different flux ratios $F_{\text {thbr }} / F_{\text {bbdy }}$ of 0.6 and 0.02 , for the primary and secondary region, respectively.

The luminosity observed during the faint phase in 1993 is a factor of 5 lower compared to primary accretion sites, and emission from that accretion area shows a marked soft X-ray deficiency. Such an effect has already been noted in the ROSAT spectra of polars observed in intermediate and low states (Ramsay et al. 1995), and is likely caused by the dominance of cyclotron cooling in low accretion rate plasmas.

The dip phase is accompanied by a marked increase of the hardness ratio in both PSPC pointings. In 1992, when the statistics were low, the mean $H R 1$ was roughly zero. For the longer PSPC pointing where the dip was only total for a short interval, the $H R 1$ also dropped to zero at the time of flux minimum, but then showed a correlated decrease as the X-ray count rate recovered to the pre-dip value. We model the change of the $H R 1$ under the assumption that it is caused by the absorption of neutral, cold matter. For this purpose we adopted a blackbody/thermal bremsstrahlung model with fixed $k T_{\mathrm{bb}}=25 \mathrm{eV}$ and $k T_{\mathrm{br}}=20 \mathrm{keV}$ for the intrinsic X-ray source, and set their normalizations according to the count rate and hardness ratio observed right after the dip egress. We then determined what absorbing column is necessary to reduce the intrinsic X-ray flux to the observed values, and computed the corresponding change of $H R 1$. As shown in Fig. 3 (right) the cold absorption model reproduces the $H R 1$ variation except for the lowest count rate bins, where the observed $H R 1=0$ is significantly softer than the predicted value of 1 . This difference might indicate a more complex absorbing medium such as partial covering or a warm absorber, or is simply due to a change of the intrinsic X-ray spectrum. The column densities within the accretion stream as derived from our modelling range from $1.1 \times 10^{20} \mathrm{~cm}^{-2}$ at the start and end of the dip to $5 \times 10^{21} \mathrm{~cm}^{-2}$ in the most opaque parts.

\section{Optical photometry}

RX J1846 was monitored during 17 nights between 1994 and 1998 utilizing telescopes at Sonneberg Observatory, 
the Astrophysical Institute Potsdam at Babelsberg (both Germany) and the Observatorio Astronomico Nacional, (Mexico) at San Pedro Martir.

These telescopes have apertures of $0.6 \mathrm{~m}, 0.7 \mathrm{~m}$ and $1.5 \mathrm{~m}$, respectively, and were equipped with CCD detectors. Exposures varied from $30 \mathrm{~s}$ to $600 \mathrm{~s}$ and were mainly taken without filters. Differential magnitudes have been computed with respect to star A (Fig. 1), which has a $R$ magnitude of 15.6 as measured in the USNO catalog. The images itself were processed using the profile-fitting scheme of the DoРнот reduction package (Mateo \& Schechter 1989). The combined log of all observations is given in Table 1.

\subsection{Orbital variations}

Although RX J1846 displayed a large variety of light curve morphologies during the four years of monitoring, it showed one persistent photometric feature: a steep decline of at least half a magnitude. This has been used for a period search based on the analysis-of-variance method (SchwarzenbergCerny 1989) and a least squares calculation applied to the barycentric timings of the end of the steep decline (see Table 4). The resulting periodograms (Fig. 5) show only one significant periodicity at $128.7 \mathrm{~min}$. The times of the steep end of the bright hump follow a linear ephemeris given by

$$
\begin{array}{r}
T_{\text {end }}(\mathrm{BJD})=2449625.5502(26) \\
+E \times 0.089386876(10)
\end{array}
$$

with the numbers in brackets giving the uncertainty in the last digits. The deviation of the observed times with respect to Eq. (1) can be as large as $10 \mathrm{~min}$, and is likely caused by an azimuthal drift of the primary accretion spot of the order of $30^{\circ}$. In Fig. 4 we present the set of all available light curves with more than one orbit coverage, folded over the ephemeris derived above. Apparently, the system dropped from a high state in 1994/1995 into an intermediate state in 1997/1998. This drop of the overall brightness of $\sim 1$ mag was accompanied by a drastic change in the light curve morphology.

During the intermediate state (Fig. 4, right) the light curves are dominated by one active pole, self-eclipsed by the body of the white dwarf, evident by a $\sim 1$ mag brightening every $128 \mathrm{~min}$. The duration of the bright phase was 0.4 and 0.45 on January 25 and March 24 1998, placing the primary pole to the farther hemisphere of the white dwarf. At these occasions the rise to maximum occurred just within a few minutes, and the flux remained more or less constant throughout the bright phase, showing no signs of cyclotron beaming. We also note a systematic brightening during faint phase by $\sim 0.5 \mathrm{mag}$, a feature which can be associated with the presence of a fainter secondary accretion region. The shape of the bright phase as well as the behaviour during the faint phase are very similar to the well-studied self-eclipsing polar ST LMi (Cropper 1986), which has an inclination of $i=56^{\circ}$ and a colatitude $\beta=134^{\circ}$. On August 51998 we observed a major restructuring of the main accretion region. The bright phase was prolonged to 0.65 of the orbit and was highly asymmetric with a very slow rise to maximum for almost the entire time followed by a steep

\begin{tabular}{|c|c|c|c|}
\hline $\begin{array}{c}T_{\text {end }} \\
(\text { HJD } 2400000+)\end{array}$ & $\begin{array}{l}\Delta T_{\text {end }} \\
\quad(10\end{array}$ & O-C & Cycle \\
\hline 49617.5070 & 25 & 16 & -90 \\
\hline 49618.4032 & 25 & 39 & -80 \\
\hline 49619.3817 & 25 & -9 & -69 \\
\hline 49623.4024 & 20 & -26 & -24 \\
\hline 49625.4602 & 15 & -7 & -1 \\
\hline 49625.5493 & 15 & -9 & 0 \\
\hline 49840.8845 & 20 & 15 & 2409 \\
\hline 49842.9370 & 20 & -18 & 2432 \\
\hline 50015.3664 & 28 & 5 & 4361 \\
\hline 50750.2970 & 20 & -68 & 12583 \\
\hline 50839.6037 & 14 & 25 & 13582 \\
\hline 50839.6936 & 14 & 29 & 13583 \\
\hline 50897.5253 & 7 & 14 & 14230 \\
\hline 50897.6131 & 7 & -2 & 14231 \\
\hline 51031.5133 & 7 & -13 & 15729 \\
\hline 51081.4827 & 7 & 9 & 16288 \\
\hline
\end{tabular}

Table 4. Barycentric timings of the end of bright phase.

decline. This asymmetry can be understood in terms of an accretion arc. The orientation of this arc has to be such, that it is orthogonal to the limb of the white dwarf during rise, and parallel when it disappears behind it.

At the time of the optical high state the orbital variation is much less pronounced, but still marked by a 0.5 mag drop at phase $\phi=0$ due to the disappearance of the primary accretion region. Other notable features are the double humped light curve on September 29, 1994, and the sharp dips observed in May 1995 at phase $\phi=0.7$. The latter are coinciding in phase with the X-ray dip seen in most of the ROSAT observations, probably caused by photoelectrical absorption by material in the accretion stream. Although less evident than the $\mathrm{X}$-ray light curves, we interpret the enhanced optical flux between $\phi=0 . .0 .4$ as the manifestation of simultaneous accretion onto a second pole.

\subsection{Long-term behaviour}

In order to inspect the frequency of the brightness and accretion mode changes we compiled all available measurements of RX J1846 (Table 5). For the single, not phase-resolved measurements we assigned the possible accretion mode depending on the brightness at phase $\phi=0.3$, if available. We find in our data at least six alterations between single and two-pole accretion between 1990 and 1999. The amount of data is yet insufficient to detect any possible periodicity of these changes. It is noteworthy that the system was never observed in the single accretion state with the secondary pole being active.

The time scale of the change is constrained by the observations in September/October 1994, when RX J1846 dropped into an intermediate state within at most two weeks. An indication that such changes might be much faster comes from the light curve obtained on Sep. 24, 1998. At that occasion the 


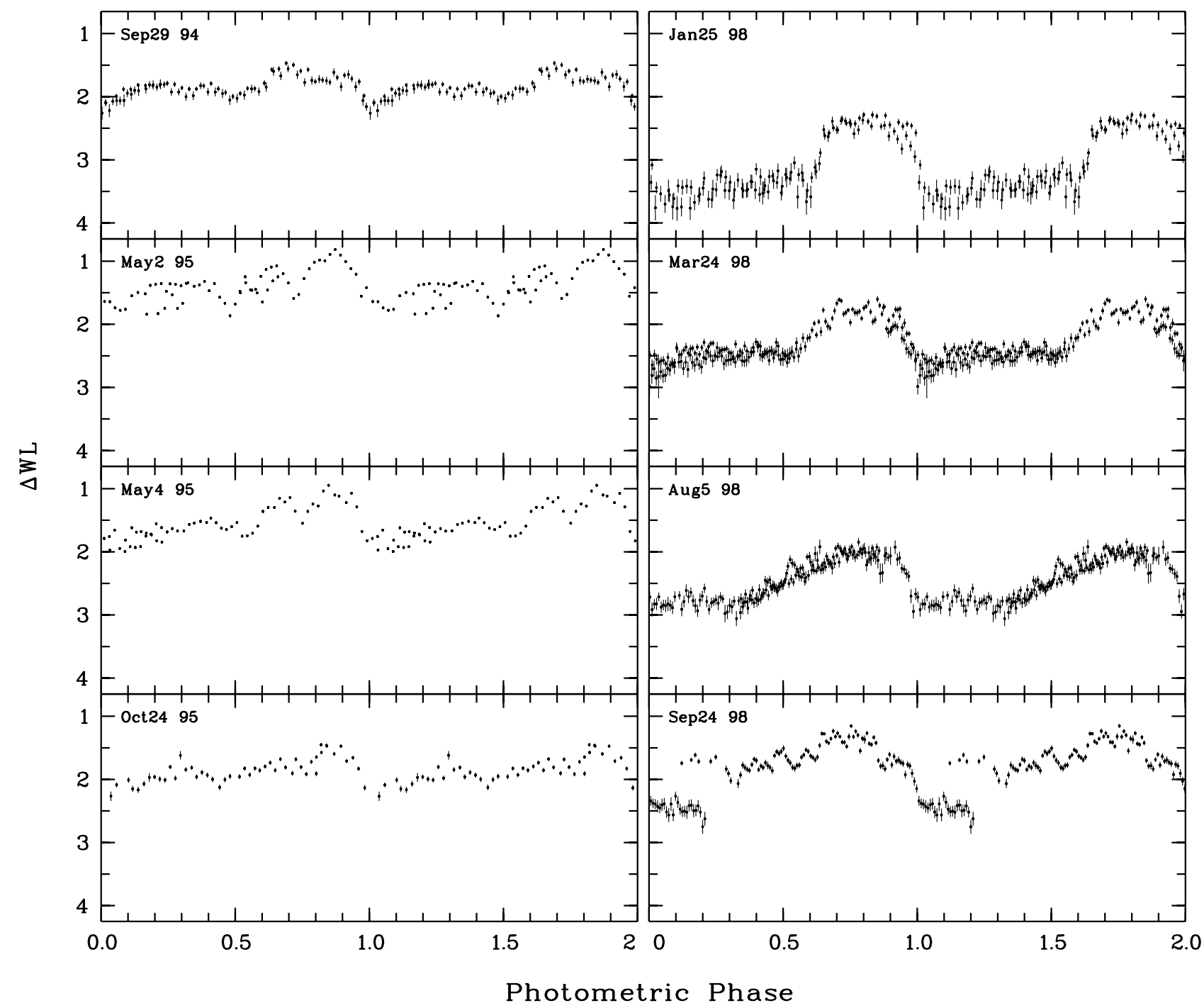

Fig. 4. Optical light curves of RX J1846 plotted as a function of ephemeris Eq. (1). Data are plotted twice for clarity.

system's brightness at phase 0.3 faded from a level typical for the high state by 0.8 mag within two consecutive orbital cycles.

\section{Optical spectroscopy}

First spectroscopic identification observations were performed on October 1, 1992 with the $3.5 \mathrm{~m}$ telescope at Calar Alto, Spain. We used the Cassegrain spectrograph equipped with a RCA CCD as detector covering the optical wavelength range from 3800-7100 $\AA$. The observation was obtained under stable photometric conditions and accompanied by measurements of the standard star Feige 110, which was used to calibrate the flux with an estimated accuracy of $\sim 20 \%$ (using standard MIDAs procedures). By convolving the original spectrum with functions representing the $B V R$ bandpasses, we arrive at $B=18.6$, $V=18.7$ and $R=18.4 \mathrm{mag}$ for $\mathrm{RX} \mathrm{J} 1846$. The identification exposure on October 1, 1992 lasted one hour corresponding to approximately half of the binary orbit, and was centered on HJD 2448897.4271 which corresponds to $\phi=0.25$ of the ephemeris given by Eq. (1). Thus, the spectrum covers the complete faint phase observed in the one-pole accretion mode, and the magnitudes derived above are consistent with that found in the intermediate accretion state. The original spectrum is shown in Fig. 6. It is dominated by intense emission lines of the Balmer series, He II $\lambda 4686 \AA$, and He I superimposed on a flat continuum. The inverted Balmer decrement and the strength of the He II $\lambda 4686 \AA$ line point to a magnetic CV classification.

\subsection{Cyclotron spectroscopy}

Further phase-resolved low-resolution spectroscopy was obtained on June 19, 1999 with the $6 \mathrm{~m}$ SAO telescope at Zelenchukskaja, Russia. The SP-124 spectrograph with a $300 \mathrm{l} / \mathrm{mm}$ grating was used to obtain spectra in the $3400-7900 \AA$ range with $9 \AA(F W H M)$ resolution. An exposure time of $900 \mathrm{~s}$ was necessary to reach a reasonable signalto-noise ratio. We obtained 14 spectra thus covering the orbital period twice. The $R$-band light curves derived from the spectra showed a brightening from $17^{\mathrm{m}}$ to $16^{\mathrm{m}}$ for half of the orbit, very similar to that observed in the one-pole accretion mode in $1997 / 98$. 


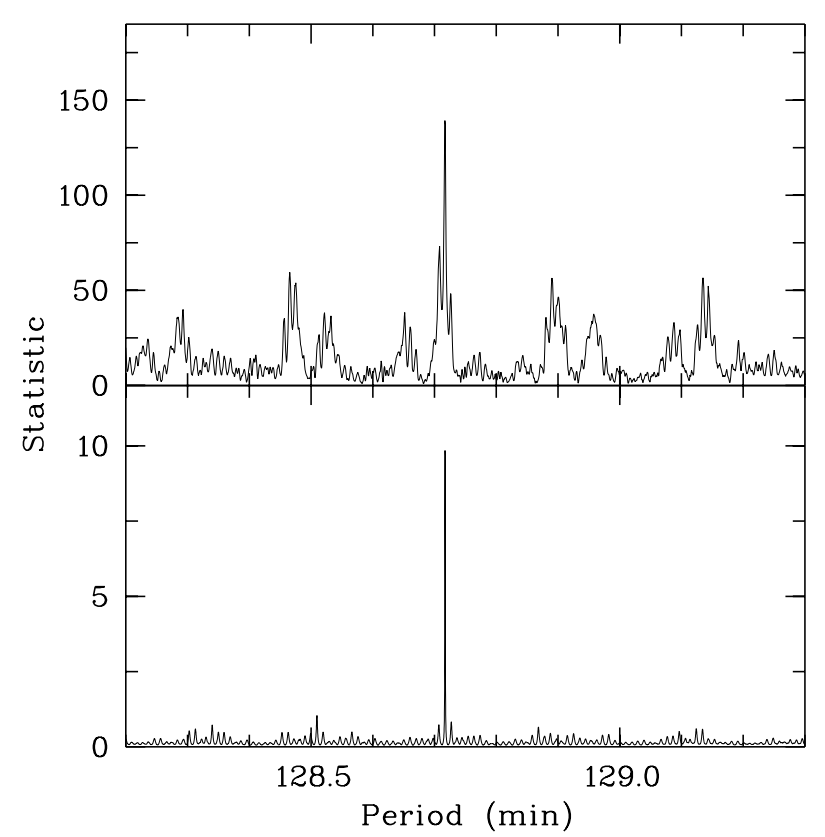

Fig. 5. Periodograms based on the analysis-of-variance method computed from all available optical photometry (upper panel) and leastsquares method applied to the timings of the end of bright phase given in Table 4 (lower panel). Likely periods appear as maxima.

In order to extract the cyclotron component from the primary accretion region we took the difference spectrum of the bright $(\phi>0.45)$ and faint $(\phi<0.45)$ phase as shown in the lower panel of Fig. 7. It is characterized by a very red (zero flux below $4000 \AA$ ) and smooth continuum peaking at $\lambda=6700 \AA$, and bears strong resemblance with that of other low field polars like BL Hyi (15 MG; Schwope et al. 1995a), EP Dra (16 MG; Schwope \& Mengel 1997) or CP Tuc (Thomas \& Reinsch 1996).

In this low magnetic field strength regime only the high harmonic tail $(m>10)$ is accessible in the optical, where the individual harmonics are broadened and blended into a quasicontinuum. The peak of the cyclotron flux corresponds to the turnover between the optically thick part of the spectrum represented by the Rayleigh-Jeans tail of a black body, and the optically thin part, which is steeply declining towards smaller wavelengths due to the $F \sim \lambda^{2}$ dependence of the cyclotron absorption coefficients. The harmonic number at which the cyclotron spectrum becomes optically thin depends on the plasma temperature and the optical depth parameter $\Lambda$ and can be used for an estimate of the magnetic field strength $B$ in the accretion region.

Setting the yet unknown plasma temperature to a value of $10 \mathrm{keV}$ typically found in polars we computed the expected wavelength of turnover for different values of $\Lambda$ and $B$ using cyclotron absorption coefficients according to Chanmugam \& Dulk (1981). For the typical range of depth parameters $\log (\Lambda)=6 . .8$ observed in high accretion state polars the peak of the cyclotron flux at $\lambda=6700 \AA$ would correspond to a magnetic field strength in the primary accretion region of RX J1846 of $B=20 . .15 \mathrm{MG}$. The integrated flux of the cyclotron component in the range $13400-7900 \AA$ is $0.6 \times 10^{-12} \mathrm{erg} \mathrm{cm}^{-2} \mathrm{~s}^{-1}$.
Table 5. Long term variations of RX J1846.

\begin{tabular}{cccc}
\hline \hline Date & Type & Brightness $^{1}$ & Mode \\
\hline Oct. 90 & $\mathrm{X}$ & 0.0 & one-pole \\
Oct. 91 & POSSII & $\sim 20^{\mathrm{m}}$ & $?$ \\
Jun. 92 & $\mathrm{X}$ & 0.42 & two-pole \\
Oct. 92 & Spec & $18 \mathrm{~m} .4$ & one-pole \\
Sep. 93 & $\mathrm{X}$ & 0.05 & one-pole \\
Sep. 94 & Phot & 17.5 & two-pole \\
Oct. 94 & Phot & 18.9 & one-pole \\
Apr. 95 & $\mathrm{X}$ & $0.2^{2}$ & $?$ \\
May 95 & Phot & 17.3 & two-pole \\
Oct. 95 & Phot & 17.6 & two-pole \\
Oct. 97 & Phot & 19.6 & one-pole \\
Jan. 98 & Phot & $19 \mathrm{~m}^{\mathrm{m}} 1$ & one-pole \\
Mar. 98 & Phot & 18.1 & one-pole \\
Aug. 98 & Phot & $18^{\mathrm{m}} .4$ & one-pole \\
Sep. 98 & Phot & & switching \\
Sep. 99 & Spec & 17.2 & one-pole \\
\hline
\end{tabular}

${ }^{1}$ Brightness between $\phi=0 . .0 .4$ X-ray: PSCP countrate; Optical photometry: magnitudes have been calculated from differential white light measurements and the USNO A.2 $R$-band magnitudes of the comparison star. ${ }^{2}$ HRI countrate has been converted using a factor of 7.8 .

Accounting for a similar contribution from the infra-red not covered by our spectra the total, bolometric flux is of the same order as the X-ray bremsstrahlung component.

\subsection{A rough distance estimate}

The faint phase, intermediate state spectrum taken in October 1992 is devoid of any spectral features of the secondary star (e.g. the TiO bands at $\lambda 6159$ and $6651 \AA$ ). We estimate that the contribution of the companion is less then $10 \%$ in the $R$-band i.e. $R_{\mathrm{sec}} \gtrsim 19$. 9 . This can, in principle, be used to derive a lower limit on the distance of the system. Assuming that the secondary in RX J1846 is a dM 4.5 star, as expected for a Roche lobe filling main-sequence star at this orbital period, it should have an absolute magnitude of $M_{R}=11.89$ (Kirckpatrick \& McCarthy 1994). This leads to a distance modulus of 8.0 or a lower limit of the distance of $400 \mathrm{pc}$. We caution however, that the spectral features involved can be highly suppressed on the front side of the secondary by the strong EUV/X-ray radiation. As the viewing geometry of RX J1846 is as yet unknown, we can not exclude that a non-negligible fraction of the disturbed atmosphere visible at the time of our spectroscopy caused the absence of the TiO bands. This in turn would decrease the distance estimated above.

\section{Discussion}

RX J1846 shows all major hallmarks of a cataclysmic variable of the AM Herculis subclass: an emission line spectrum with abundant high ionisation species like He II $\lambda 4686$, and the Bowen blend, optical and X-ray light curves strongly modulated on the spin period due to the self eclipse of the primary 

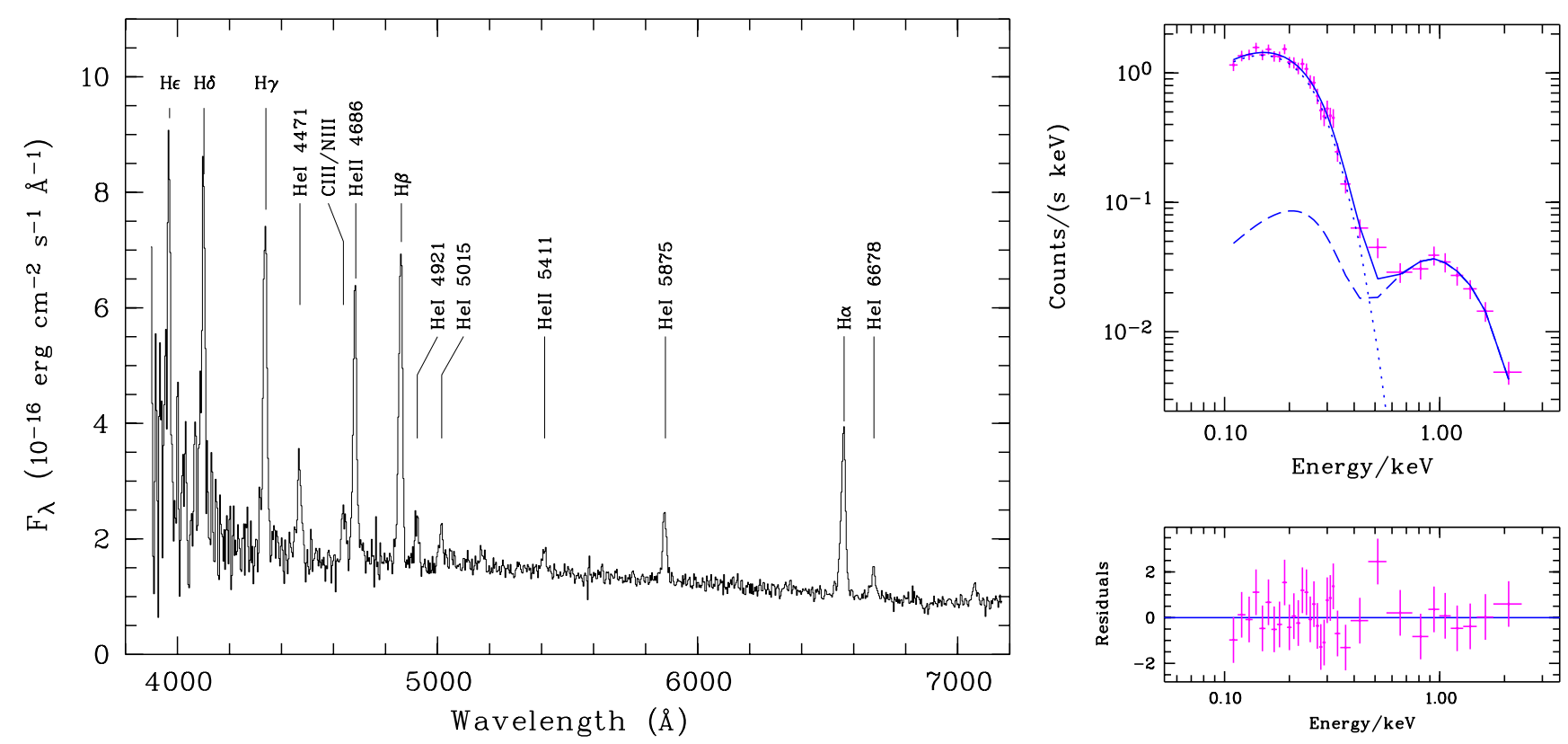

Fig. 6. Left: low resolution optical spectrum of RX J1846.9+5538 obtained on October 1, 1992. Main emission lines are indicated. Right: phase-averaged X-ray spectrum of the June 1992 PSPC observation of RX J1846 unfolded with the sum of a blackbody and a thermal bremsstrahlung spectrum with a temperature fixed at $20 \mathrm{keV}$. The lower right panel shows the residua of the fit in units of $\sigma$.

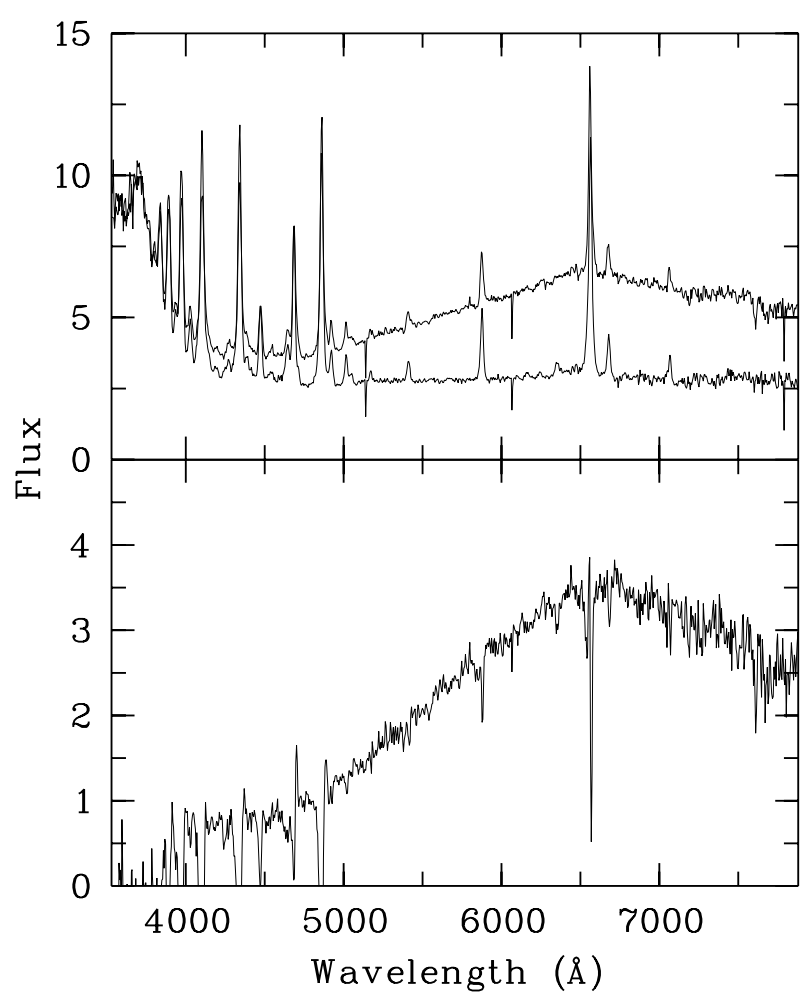

Fig. 7. Spectroscopy obtained at SAO on June 19 1999. In the upper panel averaged spectra of the bright and faint phase are displayed. The difference of those two spectra (lower panel) corresponds to the cyclotron spectrum of the primary accretion region. Flux is given in units of $10^{-16} \mathrm{erg} \mathrm{cm}^{-2} \mathrm{~s}^{-1} \AA^{-1}$.

accretion region, and a very red cyclotron spectrum from that primary accretion region, indicating a polar field strength in the range of 15-20 MG. At most occasions the X-ray light curves reveal sharp X-ray dips, likely caused by absorption within a focused accretion funnel. With an orbital period of $P=128.7$ min RX J1846 is another magnetic CV which populates the lower edge of the $2-3 \mathrm{hr} \mathrm{CV}$ period gap.

\subsection{Accretion mode changes}

The rather large body of observations available for RX J1846 reveals frequent switches between one-pole accretion and additional activity from a second, equally bright, pole. Although accretion onto a second pole has been seen in a few polars, e.g. in DP Leo (Cropper et al. 1990), VV Pup (Wickramasinghe et al. 1989) or UZ For (Schwope et al. 1990), in most cases the activity from the secondary pole is at least one order of magnitude lower compared to the primary one. Remarkable exceptions include the "anomalous" or "reversed" state of AM Her (Heise et al. 1985), which appeared to be a singular event, and the low accretion rate polar HS1023+39 which permanently accretes onto two poles having similar luminosities (Reimers et al. 1999; Schwarz et al. 2001). So far the only polar, beside RX J1846, which regularly changes between the single and two-pole accretion mode is the period-gap system QS Tel (Schwope et al. 1995a; Rosen et al. 1996, 2001).

There are two alternative mechanisms that can trigger the accretion mode changes observed in RX J1846. Firstly, it can be due to a slight asynchronism of the spin of the white dwarf compared to the orbital motion, which in the case of an oblique dipole results in a constantly changing orientation of the magnetic field with respect to the infalling gas stream. Matter can then be channeled along different field lines, possibly feeding opposite magnetic poles. While this so-called "pole switching" is believed to operate in the four known asynchronous AM Herculis systems (Campbell \& Schwope 1999), detailed 
studies indicate that the mass exchange might be more complicated. For example in CD Ind, the only asynchronous polar with known accretion geometry, one primary pole dominates in the light curves for a large fraction of the beat cycle (Ramsay et al. 2000). Thus, the fact that RX J1846 does not switch between two magnetic poles but has one primary pole might still be consistent with a possible asynchronism. A more crucial test of this scenario is the requirement that the accretion mode changes should occur strictly periodically over the beat cycle, a possibility which can not yet be rejected for RX J1846 on the basis of the available data.

The second mechanism would be variations of the total mass accretion rate from the secondary star, which are commonly seen as low states in disk-less magnetic CVs. These variations are fast, and reoccur aperiodically on timescales of months to years. The working hypothesis that explains most of the observed properties invokes the blocking of the $L_{1}$ point by starspots on the surface of the secondary star as proposed by Livio \& Pringle (1994). As the total mass accretion rate increases, the ram pressure $(\rho v)$ increases in the stream, leading to a deeper penetration of the ballistic mass stream into the white dwarf's magnetosphere. Possibly the stream will then connect to field lines which can also feed the less favoured pole. By now, all observed two-pole accretion states in RX J1846 have been related to epochs of enhanced total brightness in the optical and X-ray, thus indicating an increase of the mass accretion rate. This correlation is so far the strongest argument in favour of the above picture. If the pressure balance relation holds, the effective magnetospheric radius at which matter is controlled by the magnetic field would scale with the mass accretion rate with $r_{\text {mag }} \propto \dot{M}^{-2 / 7}$. The brightening of the total X-ray flux by a factor of $\sim 2$ in the two-pole state implies that the accretion rate must have roughly doubled at that epoch. The corresponding reduction of the magnetospheric radius would then be only of the order of $20 \%$, probably not enough to reach the less favoured magnetic pole in the case of the standard field orientation seen in AM Herculis binaries with an azimuthal angle of $\psi=45^{\circ}$ (see Cropper 1988). Indeed, long-term X-ray monitoring of the eclipsing polar HU Aqr (Schwope et al. 2001) through different accretion states revealed only a moderate shift of the stagnation region of only $30^{\circ}$ as the accretion rate varied by a factor of 40 . Thus, in addition to the accretion rate changes, a special field geometry is possibly required to facilitate the frequent accretion mode changes in RX J1846.

Both accretion regions emit approximately the same accretion luminosities of $L_{\mathrm{X}}=7 \times 10^{31}(\mathrm{D} / 400 \mathrm{pc})^{2} \mathrm{erg} / \mathrm{s}$, thus receiving quite similar mass accretion rates. However, the contributions from the hot thermal plasma, emitted as hard X-rays, and reprocessed radiation from the white dwarf emitted as a blackbody in the soft X-ray band is remarkably different in both spots. While the energy balance of the primary region is in agreement with that of the standard shock model (Lamb \& Masters 1979), a substantial soft X-ray excess is found for the secondary spot. This violation has been theoretically explained in terms of "blobby" accretion of high $\dot{m}\left(>30 \mathrm{~g} \mathrm{~cm}^{-2} \mathrm{~s}^{-1}\right)$ material buried below the surface of the white dwarf (Kuijpers \& Pringle 1982). Observationally, a strong correlation between the magnetic field strength and the softening of the X-ray spectra has been found (Beuermann \& Schwope 1994) from a comparative study of ROSAT data. Probably two different processes are involved, one increasing the soft X-ray output and the other lowering the hard X-ray component, both changing the energy balance as observed: firstly, the specific mass accretion rate in the accretion region will be higher for material which travels further along converging field lines, and was consequently coupled at larger magnetospheric radii. Since this quantity is directly related to the surface field strength, for high $B$ systems the fraction of high $\dot{m}$ material will be larger on average, shifting the energy balance towards the reprocessed blackbody component (Beuermann 1998). Secondly, for the low $\dot{m}$ part of the material, bremsstrahlung radiation will be suppressed due to the dominance of cyclotron cooling in the case of an increased magnetic field in the post shock plasma (Woelk \& Beuermann 1996).

Adopting this picture for RX J1846, the X-ray softness of the secondary spot would be the consequence of a higher field strength of $\sim 30 \mathrm{MG}$ at this pole. Correspondingly, the energy balance of the primary region would imply a lower field strength of $\sim 14 \mathrm{MG}$, in agreement with the value derived from the cyclotron spectroscopy. As in the six polars with field measurements available for both magnetic poles, the more active pole in RX J1846 would be the one with the lower field strength.

\subsection{Accretion geometry}

A special geometry of the mass transfer might foster the drastic variations of the accretion modes observed in RX J1846. For example, an orientation where the dipolar axis is perpendicular to the infalling stream would equally favour both poles. However, this is difficult to verify given the as yet unknown position of the secondary star with respect to the accretion regions, and the wide range of possible inclinations $\left(i \leq 70^{\circ}\right)$. There is a strong indication that the primary accretion region is located at the lower hemisphere of the white dwarf $\left(\beta_{1}>90^{\circ}\right)$, from the duration of the faint phase $(\gamma>0.5)$ measured at some occasions in the optical and X-ray. Estimates for the secondary accretion region are much less constrained due to the insufficient phase coverage the of June 1992 observation and the overlapping visibility of both regions around $\phi \sim 0.5$. Using the short duration of the supersoft emission $(\Delta \phi \sim 0.3)$ as a measure would place this spot on the lower hemisphere of the white dwarf, too.

Both accretion spots are separated in azimuth by $160^{\circ}$, since the two bright phases are centered at $\phi_{1}=0.75$ and $\phi_{2}=0.2$. Due to the finite coupling radius the accretion regions are likely to be offset from the magnetic pole by $\alpha \sim 10^{\circ}$. Therefore, the measured angular separation is consistent with both spots accreting via one closed field line of a dipole field. A geometry that can accomplish the all above constraints would imply a magnetic field axis inclined into the orbital plane.

Another puzzling question concerns the accretion stream dip and its relation to the two accretion regions. As the azimuth of the stream dip and the primary spot are approximately the same, one would naively expect that the absorbing matter 
is transferred to the primary accretion region. This contradicts the condition for a stream occultation $i \geq \beta+\alpha$ given the primary spots colatitude $\beta_{1} \geq 90^{\circ}$. The above conflict could be circumvented if one allows for a possible vertical extent of the ballistic part of the stream and the stagnation region, an explanation proposed to explain a partial stream dip observed in the extreme UV light curves of the high inclination polar UZ For (Warren et al. 1995). It is not clear how far this is viable for the case of RX J1846, where the absorbing material is denser and must extend much further above the orbital plane in order to produce a dip at the given lower inclination.

The competing scenario, absorption by material channeled to a second pole in the upper hemisphere, would lead to the occultation of X-rays from the primary region for a wide range of possible stream geometries. Such a view is also supported by the putative correlation of the density of the stream with the respective activity from the secondary accretion region: no stream dip is observed when the faint phase emission is zero (ROSAT survey), a mildly dense $\operatorname{dip}\left(N_{\mathrm{H}} \sim 10^{21} \mathrm{~cm}^{-2}\right)$ for low faint phase emission (Sep. 93), and a much denser dip in the case of obvious two-pole accretion (Jul. 92). Further detailed studies have to show whether the footpoint of such a stream trajectory can be reconciled with the geometrical constraints for the secondary pole, namely the azimuthal separation to the primary spot (which would place it on the opposite side of the white dwarf) and a high colatitude far from the white dwarf rotational axis.

Acknowledgements. RS would like to thank A. D. Schwope for helpful comments on the manuscript. RS and JG are supported by the Deutsches Zentrum für Luft- und Raumfahrt (DLR) GmbH under contract No. FKZ 50 QQ 96023 and 50 OR 91068. GHT acknowledges support by grants from DGAPA IN109195 and CONACYT No 25454-E. The ROSAT project was supported by the German Bundesministerium für Bildung, Wissenschaft, Forschung und Technologie (BMBF/DLR) and the Max-Planck-Society.

Part of Fig. 1 is based on photographic data of the National Geographic Society - Palomar Observatory Sky Survey (NGS-POSS) obtained using the Oschin Telescope on Palomar Mountain. The NGSPOSS was funded by a grant from the National Geographic Society to the California Institute of Technology. The plates were processed into the present compressed digital form with their permission. The Digitized Sky Survey was produced at the Space Telescope Science Institute under US Government grant NAG W-2166.

\section{References}

Beuermann, K., \& Schwope, A. D. 1994, ASP Conf. Ser., 56, 119 Beuermann, K., \& Burwitz, V. 1995, ASP Conf. Ser., 85, 99
Beuermann, K. 1998, Perspectives in High Energy Astronomy \& Astrophysics, ed. P. C. Agrawal, \& P. R. Vishwanath (Universities Press, India), 100

Burwitz, V., Reinsch, K., Schwope, A. D., et al. 1996, A\&A, 305, 507

Campbell, C. G., \& Schwope, A. D. 1998, A\&A, 343, 132

Chanmugam, G., \& Dulk, G. A. 1981, ApJ, 244, 569

Cropper, M. 1986, MNRAS, 222, 853

Cropper, M. 1988, MNRAS, 231, 597

Cropper, M., Mason, K. O., \& Mukai, K. 1988, MNRAS, 243, 565

Dickey, J. M., \& Lockman, F. J. 1990, ARA\&A, 28, 215

Greiner, J., Remillard, R., \& Motch, C. 1995, in Cataclysmic Variables, ed. A. Bianchini, M. Della Valle, \& M. Orio, ASSL, 205, 161

Greiner, J. 1996, in Supersoft X-ray Sources, Lecture Notes in Physics 472, ed. J. Greiner (Springer), 285

Greiner, J., Remillard, R., \& Motch, C. 1998, A\&A, 336, 191

Greiner, J., Schwarz, R., \& Wenzel, W. 1998, MNRAS, 296, 437

Heise, J., Brinkman, A. C., Gronenschild, E., et al. 1985, A\&A, 148, L14

Kirkpatrick, J. D., \& McCarthy, D. W. 1994, AJ, 107, 333

Kuijpers, J., \& Pringle, J. E. 1982, A\&A, 114, L4

Livio, M., \& Pringle, J. E. 1994, ApJ, 427, 956

Lamb, D., \& Masters, A. R. 1979, ApJ, 222, 263

Mateo, M., \& Schechter, P. 1989, 1st ESO/ST-ECF Data Analysis Workshop, ed. P. J. Grosbol, F. Murtagh, \& R. H. Warmels, 69

Ramsay, G., Cropper, M., \& Mason, K. O. 1995, MNRAS, 276, 1382

Ramsay, G., Potter, S., Cropper, M., Buckley, D. A. H., \& Harrop-Allin, M. K. 2000, MNRAS, 316, 225

Reimers, D., Hagen, H.-J., \& Hopp, U. 1999, A\&A, 343, 157

Rosen, S., Rainger, J. F., Burleigh, M. R., et al. 1996, MNRAS, 280, 1121

Rosen, S., Mittaz, J. P. D., Buckley, D. A., et al. 2001, MNRAS, 322, 631

Schwarz, R., Schwope, A. D., \& Staude, A. 2001, A\&A, 374, 189

Schwarzenberg-Czerny, A. 1989, MNRAS, 241, 153

Schwope, A. D., Beuermann, K., \& Thomas, H.-C. 1990, A\&A, 230, 120

Schwope, A. D., Thomas, H., Beuermann, K., et al. 1995, A\&A, 293, 764

Schwope, A. D., Beuermann, K., \& Jordan, S. 1995, A\&A, 301, 447

Schwope, A. D., \& Mengel, S. 1997, AN, 318, 25

Schwope, A. D., Schwarz, R., Sirk, M., \& Howell, S. B. 2001, A\&A, 375,419

Thomas, H.-C., \& Reinsch, K. 1996, A\&A, 315, L1

Warner, B. 1995, Cataclysmic Variable Stars (Cambridge Univ. Press, Cambridge)

Warren, J. K., Sirk, M. M., \& Vallegra, J. V. 1995, ApJ, 445, 909

Wickramasinghe, D. T., Ferrario, L., \& Bailey, J. 1989, ApJ, 342, L35

Woelk, U., \& Beuermann, K. 1996, A\&A, 306, 232

Zimmermann, H. U., Becker, W., Belloni, T., et al. 1994, MPE report, 257 